\title{
ON A GENERALIZATION OF THE ARC-SINE LAW
}

\author{
BY LAJOS TAKÁCS
}

\section{Case Western Reserve University}

This paper deals with the distribution function of the sojourn time of Brownian motion with drift. By some recent results of J. Akahori and A. Dassios this distribution function can be expressed in the form of a double integral. In this paper it is shown that the distribution function of the sojourn time can be expressed simply as a single integral. The result obtained is a generalization of the arc-sine law of Paul Lévy.

1. Introduction. Let $\{\xi(u), u \geq 0\}$ be a standard Brownian motion process. We have $\mathbf{P}\{\xi(u) \leq x\}=\Phi(x / \sqrt{u})$ for $u>0$, where

$$
\Phi(x)=\frac{1}{\sqrt{2 \pi}} \int_{-\infty}^{x} \exp \left(-\frac{u^{2}}{2}\right) d u
$$

is the normal distribution function. We shall consider the process $\{\sigma \xi(u)+$ $m u, u \geq 0\}$, where $\sigma>0$ and $m$ is a real number. We use the notation $\delta(A)$ for the indicator variable of an event $A$; that is, $\delta(A)=1$ if $A$ occurs and $\delta(A)=0$ if $A$ does not occur. Define

$$
\tau(\alpha, m, \sigma, t)=\frac{1}{t} \int_{0}^{t} \delta(\sigma \xi(u)+m u \leq \alpha) d u
$$

for $t>0$; that is, $t \tau(\alpha, m, \sigma, t)$ is the sojourn time of the process $\{\sigma \xi(u)+$ $m u, u \geq 0\}$ spent in the set $(-\infty, \alpha]$ in the time interval $(0, t)$.

We also define

$$
\gamma(x, m, \sigma, t)=\inf \left\{\alpha: \frac{1}{t} \int_{0}^{t} \delta(\sigma \xi(u)+m u \leq \alpha) d u>x\right\}
$$

for $t>0$; and $0<x<1$; that is, $\{\gamma(x, m, \sigma, t), 0<x<1\}$ is the inverse process of $\{\tau(\alpha, m, \sigma, t),-\infty<\alpha<\infty\}$.

Recently Akahori [1] and Dassios [4] determined the distributions of $\tau(\alpha, m, \sigma, t)$ and $\gamma(x, m, \sigma, t)$. The distribution functions of both random variables can be expressed by their results in the form of a double integral. In this paper it is shown that by using an elementary random walk approach, these distribution functions can be expressed in the form of a single integral. The result obtained can be interpreted as a generalization of the arc-sine law of Lévy [7, 8].

2. The distribution of $\tau(\alpha, m, \sigma, t)$. First, we observe that

$$
\{\gamma(x, m, \sigma, t)>\alpha\} \equiv\{\tau(\alpha, m, \sigma, t) \leq x\}
$$

Received August 1995.

AMS 1991 subject classifications. Primary 60J15, 60J55, 60J65.

Key words and phrases. Random walk, Brownian motion, sojourn time, distribution function. 
for $0<x<1$ and $-\infty<\alpha<\infty$. Thus the problem of finding the distribution of (3) can be reduced to the problem of finding the distribution of (2).

Next, we observe that it is sufficient to consider only the particular case where $\alpha \geq 0, \sigma=1$ and $t=1$. The general case can be reduced to this particular case by linear transformations. Since for any $t>0$, the two processes $\{\xi(u t), u \geq 0\}$ and $\{\sqrt{t} \xi(u), u \geq 0\}$ have the same finite dimensional distributions, we have

$$
\tau(\alpha, m, \sigma, t) \sim \tau(\alpha / \sigma \sqrt{t}, m \sqrt{t} / \sigma, 1,1)
$$

for $t>0$, where the symbol $\sim$ means that the random variables on both sides of (5) have the same distribution. Moreover, by symmetry we have

$$
\tau(\alpha, m, \sigma, t) \sim 1-\tau(-\alpha,-m, \sigma, t)
$$

for $t>0$. Accordingly, if we know the distribution of

$$
\tau(\alpha, m)=\tau(\alpha, m, 1,1)
$$

for $\alpha \geq 0$ and $m \in(-\infty, \infty)$, then the distribution of (2) is determined by (5) and (6).

In a similar way we obtain that

$$
\gamma(x, m, \sigma, t) \sim \gamma(x, m \sqrt{t} / \sigma, 1,1) \sigma \sqrt{t}
$$

for $t>0$ and $0<x<1$. Thus, if we know the distribution of

$$
\gamma(x, m)=\gamma(x, m, 1,1)
$$

for $0<x<1$, then the distribution of (3) is determined by (8).

By (4) we have

$$
\mathbf{P}\{\gamma(x, m) \leq \alpha\}=\mathbf{P}\{\tau(\alpha, m)>x\}
$$

for $0<x<1$, and by (6)

$$
\tau(\alpha, m) \sim 1-\tau(-\alpha,-m) .
$$

The distribution functions of (2) and (3) are completely determined by the following theorem.

THEOREM 1. If $\alpha \geq 0$ and $m \in(-\infty, \infty)$, then

$$
\begin{aligned}
\mathbf{P}\{\tau(\alpha, m) \leq x\}=2 \int_{0}^{x} & {\left[\frac{\varphi(m \sqrt{1-u})}{\sqrt{1-u}}+m \Phi(m \sqrt{1-u})\right] } \\
\times & {\left[\varphi\left(\frac{\alpha-m u}{\sqrt{u}}\right) \frac{1}{\sqrt{u}}-m e^{2 m \alpha} \Phi\left(\frac{-\alpha-m u}{\sqrt{u}}\right)\right] d u }
\end{aligned}
$$

for $0 \leq x<1$, where

$$
\varphi(x)=\frac{1}{\sqrt{2 \pi}} \exp \left(-\frac{x^{2}}{2}\right)
$$


is the normal density function and

$$
\mathbf{P}\{\tau(\alpha, m)=1\}=\Phi(\alpha-m)-e^{2 m \alpha} \Phi(-\alpha-m),
$$

where $\Phi(x)$ is defined by (1).

See Section 4 for the proof.

We note that if in Theorem $1, m=0$, then we obtain that

$$
\mathbf{P}\{\tau(\alpha, 0) \leq x\}=\frac{1}{\pi} \int_{0}^{x} \frac{\exp \left(-\alpha^{2} / 2 u\right)}{\sqrt{u(1-u)}} d u
$$

for $0 \leq x<1$ and

$$
\mathbf{P}\{\tau(\alpha, 0)=1\}=2 \Phi(\alpha)-1 .
$$

If $\alpha=0$ and $m=0$, then by Theorem 1 ,

$$
\mathbf{P}\{\tau(0,0) \leq x\}=\frac{2}{\pi} \arcsin \sqrt{x}
$$

for $0 \leq x \leq 1$. This last formula was found by Lévy ([7]; [8], page 303) in 1939 and is called the arc-sine law. See also [10]. The more general result (15) was also found by Lévy ([8], page 326), but in a form more complicated than (15). The above form is given by Yor [11]. As we have already mentioned, the distributions of (2) and (3) were determined in 1995 by Akahori ([1], Theorem 1.1) and Dassios ([4], Theorem 1).

3. A sojourn time problem for random walk. Let us suppose that $\left\{\xi_{r}, r \geq 1\right\}$ is a sequence of independent and identically distributed random variables for which

$$
\mathbf{P}\left\{\xi_{r}=1\right\}=p \quad \text { and } \quad \mathbf{P}\left\{\xi_{r}=-1\right\}=q,
$$

where $p>0, q>0$ and $p+q=1$. Define $\zeta_{r}=\xi_{1}+\xi_{2}+\cdots+\xi_{r}$ for $r \geq 1$ and $\zeta_{0}=0$. Then the sequence $\left\{\zeta_{r}, r \geq 0\right\}$ describes a random walk on the real line. Let us define $\Delta_{n}(k)$ as the number of subscripts $r=1,2, \ldots, n$ for which $\zeta_{r}>k$ and write $\Delta_{n}(0)=\Delta_{n}$; that is, $\Delta_{n}$ is the number of positive elements in the sequence $\zeta_{1}, \zeta_{2}, \ldots, \zeta_{n}$. We set $\Delta_{0}=0$. Furthermore, let us define $\rho(k)$ as the first passage time through $k$; that is,

$$
\rho(k)=\inf \left\{r: \zeta_{r}=k \text { and } r \geq 0\right\}
$$

for $k=0, \pm 1, \pm 2, \ldots$

Our aim is to determine the distribution of the random variable $\Delta_{n}(k)$ for $n=1,2, \ldots$ and $k=0, \pm 1, \pm 2, \ldots$ It is sufficient to consider only the case where $k=0,1,2, \ldots$, because $\Delta_{n}(k)$ has the same distribution as $n-\Delta_{n}^{*}(-k-$ $1)$, where $\left\{\Delta_{n}^{*}(k)\right\}$ is defined in the same way as $\left\{\Delta_{n}(k)\right\}$ except that the roles of $p$ and $q$ are interchanged. We need the following two results. 
1. If $1 \leq k \leq n$, then

$$
\mathbf{P}\{\rho(k) \leq n\}=\mathbf{P}\left\{\zeta_{n} \geq k\right\}+\left(\frac{p}{q}\right)^{k} \mathbf{P}\left\{\zeta_{n}<-k\right\} .
$$

This can be proved simply by applying the reflection principle to the random walk $\left\{\zeta_{r}, r \geq 0\right\}$. It follows by symmetry that

$$
\mathbf{P}\{\rho(-k)=j\}=\left(\frac{q}{p}\right)^{k} \mathbf{P}\{\rho(k)=j\}
$$

for $j \geq 0$. See [9].

2. If $0 \leq j \leq n$, then

$$
\mathbf{P}\left\{\Delta_{n}=j\right\}=\mathbf{P}\left\{\Delta_{j}=j\right\} \mathbf{P}\left\{\Delta_{n-j}=0\right\} .
$$

This is a particular case of a general result of Andersen ([2], page 128; [3], page 197). Evidently,

$$
\mathbf{P}\left\{\Delta_{n}=n\right\}=p \mathbf{P}\{\rho(-1) \geq n\}=p-q \mathbf{P}\{\rho(1)<n\}
$$

and

$$
\mathbf{P}\left\{\Delta_{n}=0\right\}=\mathbf{P}\{\rho(1)>n\} .
$$

THEOREM 2. If $n \geq 1$, then

$$
\mathbf{P}\left\{\Delta_{n}(k)=0\right\}=\mathbf{P}\{\rho(k+1)>n\}
$$

for $0 \leq k \leq n$ and

$$
\mathbf{P}\left\{\Delta_{n}(k)=j\right\}=\mathbf{P}\left\{\Delta_{j}=j\right\}[\mathbf{P}\{\rho(k+1)>n-j\}-\mathbf{P}\{\rho(k)>n-j\}]
$$

for $1 \leq j \leq n-k$.

Proof. Formula (25) is trivially true. If $1 \leq j \leq n-k$, then

$$
\mathbf{P}\left\{\Delta_{n}(k)=j\right\}=\sum_{i=k}^{n-j} \mathbf{P}\{\rho(k)=i\} \mathbf{P}\left\{\Delta_{n-i}=j\right\} .
$$

To obtain (27) we take into consideration that $\Delta_{n}(k)=j$ can occur in several mutually exclusive ways: the smallest $r=1,2, \ldots, n$ for which $\zeta_{r}=k$ is $i=k, \ldots, n-j$ and $\zeta_{r}-\zeta_{i}>0$ for $j$ subscripts $i<r \leq n$. By (22) we obtain that

$$
\begin{aligned}
\mathbf{P}\left\{\Delta_{n}(k)=j\right\} & =\mathbf{P}\left\{\Delta_{j}=j\right\} \sum_{i=k}^{n-j} \mathbf{P}\{\rho(k)=i\} \mathbf{P}\left\{\Delta_{n-i-j}=0\right\} \\
& =\mathbf{P}\left\{\Delta_{j}=j\right\}[\mathbf{P}\{\rho(k+1)>n-j\}-\mathbf{P}\{\rho(k)>n-j\}],
\end{aligned}
$$

and by (23) we get (26), which was to be proved. 
In Theorem 2 the probability (26) depends only on the distributions of $\zeta_{j-1}$ and $\zeta_{n-j}$. In (26) we can write that

$$
\mathbf{P}\{\rho(1) \geq j\}=\mathbf{P}\left\{\zeta_{j-1}=0\right\}+\mathbf{P}\left\{\zeta_{j-1}=-1\right\}+\left(1-\frac{p}{q}\right) \mathbf{P}\left\{\zeta_{j-1}<-1\right\}
$$

and

$$
\begin{aligned}
\mathbf{P}\{\rho(k+1)>n-j\}-\mathbf{P}\{\rho(k)>n-j\} & \\
= & \mathbf{P}\left\{\zeta_{n-j}=k\right\}+\mathbf{P}\left\{\zeta_{n-j}=k+1\right\}-\left(1-\frac{q}{p}\right) \mathbf{P}\left\{\zeta_{n-j}=k+1\right\} \\
& +\left(1-\frac{p}{q}\right)\left(\frac{p}{q}\right)^{k} \mathbf{P}\left\{\zeta_{n-j}<-k-1\right\} .
\end{aligned}
$$

The distribution of $\zeta_{n}$ is determined by

$$
\mathbf{P}\left\{\zeta_{n}=2 j-n\right\}=\left(\begin{array}{c}
n \\
j
\end{array}\right) p^{j} q^{n-j}
$$

for $0 \leq j \leq n$.

If, in particular, $p=q=1 / 2$, then by (26) we obtain that

$$
\mathbf{P}\left\{\Delta_{n}(k)=j\right\}=\left(\begin{array}{c}
j-1 \\
{[(j-1) / 2]}
\end{array}\right)\left(\begin{array}{c}
n-j \\
{[(n-j-k) / 2]}
\end{array}\right) \frac{1}{2^{n}}
$$

for $1 \leq j \leq n-k$.

4. Proof of Theorem 1. If we suppose that

$$
p=p_{n}=\frac{1}{2}+\frac{m}{2 \sqrt{n}} \quad \text { and } \quad q=q_{n}=\frac{1}{2}-\frac{m}{2 \sqrt{n}}
$$

for $n>m^{2}$, then the process $\left\{\zeta_{[n u]} / \sqrt{n}, 0 \leq u \leq 1\right\}$ converges weakly to the process $\{\xi(u)+m u, 0 \leq u \leq 1\}$ as $n \rightarrow \infty$. Since the sojourn time $\tau(\alpha, m)$ is a continuous functional on the process $\{\xi(u)+m u, 0 \leq u \leq 1\}$, we can conclude that if $k=[\alpha \sqrt{n}]$, where $\alpha>0$, and $j=[n x]$, where $0<x<1$, then

$$
\lim _{n \rightarrow \infty} \mathbf{P}\left\{\Delta_{n}(k) \geq n-j\right\}=\mathbf{P}\{\tau(\alpha, m) \leq x\}
$$

for $\alpha>0$ and $0<x<1$. (See [5], page 456.) In (26)

$$
\lim _{n \rightarrow \infty}\left(\frac{p_{n}}{q_{n}}\right)^{k}=e^{2 m \alpha}
$$

if we use (29) and (30) and if we apply the central limit theorem and the local limit theorem to $\zeta_{n}=\xi_{1}+\xi_{2}+\cdots+\xi_{n}$, then we obtain (12). (See [6], pages 191 and 233.) By (20) and (25) we obtain that

$$
\lim _{n \rightarrow \infty} \mathbf{P}\left\{\Delta_{n}(k)=0\right\}=\mathbf{P}\{\tau(\alpha, m)=1\} .
$$

This proves (14). 


\section{REFERENCES}

[1] AKahori, J. (1995). Some formulae for a new type of path-dependent option. Ann. Appl. Probab. 5 383-388.

[2] ANDERSEN, E. S. (1953). On sums of symmetrically dependent random variables. Skand. Aktuarietidskr. 36 123-138.

[3] ANDERSEn, E. S. (1954). On fluctuations of sums of random variables. II. Math. Scand. 2 195-223.

[4] Dassios, A. (1995). The distribution of the quantile of a Brownian motion with drift and the pricing of related path-dependent options. Ann. Appl. Probab. 5 389-398.

[5] Gikhman, I. I. and SkorokHoD, A. V. (1969). Introduction to the Theory of Random Processes. W. B. Saunders, Philadelphia.

[6] Gnedenko, B. V. and Kolmogorov, A. N. (1954). Limit Distributions for Sums of Independent Random Variables. Addison-Wesley, Cambridge, MA.

[7] LÉvy, P. (1939). Sur un problème de M. Marcinkiewicz. C. R. Acad. Sci. Paris Sér. I Math. 208 318-321; errata 208776.

[8] LÉvy, P. (1939). Sur certains processus stochastiques homogènes. Compositio Math. 7 283339

[9] TAKÁCs, L. (1979). Fluctuation problems for Bernoulli trials. SIAM Rev. 21 222-228.

[10] TAKÁcs, L. (1981). The arc sine law of Paul Lévy. In Contributions to Probability. A Collection of Papers Dedicated to Eugene Lukács (J. Gani and V. K. Rohatgi, eds.) 49-63. Academic Press, New York.

[11] Yor, M. (1995). The distribution of Brownian quantiles. J. Appl. Probab. 32 405-416.

2410 NEWBURY DRIVE

Cleveland Heights, OHio 44118

E-MAIL: lft@po.cwru.edu 